\title{
LA HIPOTENUSA DE PITÁGORAS COMO ÍNDICE DE AMETROPÍA
}

Gabriel Merchán Mendoza', María Susana Merchán Price², Jairo Emilio Mejía Argüello ${ }^{3}$

\section{Resumen}

Objetivo general. Crear un índice con base en la hipotenusa de Pitágoras para representar cualquier estado refractivo y facilitar los análisis estadísticos de los pacientes amétropes. Métodos. 3964 pacientes de una base de datos de Optiláser (Centro de cirugía refractiva en Bogotá, Colombia) del año 2014 fueron seleccionados para demostrar la aplicabilidad del índice de ametropía propuesto. Los pacientes no habían sido sometidos a ningún tipo de procedimiento quirúrgico. Otro ejemplo para ilustrar la aplicabilidad del índice de ametropía viene de la investigación Madre Canguro Integral llevada a cabo entre 2013 y 2014 en el Hospital de San Ignacio, Bogotá, en la cual 311 bebés nacidos prematuramente y en buen estado de salud fueron evaluados. Los meridanos principales de las ametropías se tomaron como catetos de un triángulo rectángulo para calcular la hipotenusa que fue tomada como índice de ametropía. Seguidamente, se determinó la correlación de Spearman entre el índice de ametropía y el ángulo visual expresado con mínimo ángulo de resolución (MAR). Resultados. La correlación de Spearman entre el índice de ametropía y el mínimo ángulo de resolución es 0,834 . Por tanto, es estadísticamente significativa $(\mathrm{p}:<0,001)$ y suficiente para validar el índice propuesto. Conclusiones. El índice de ametropía es un instrumento válido para la evaluación, manejo y análisis estadístico de las ametropías.

Palabras clave: índice de ametropía, hipotenusa de Pitágoras, meridianos principales.

${ }^{1}$ Optómetra. Grupo de investigación Charles Prentice, programa de Optometría, Fundación Universitaria del Área Andina. gabemerchan@yahoo.com

${ }^{2}$ Optómetra. Magíster en Educación. Especialista en Optometría Pediátrica. Docente Universidad de la Salle. Grupo de investigación Charles Prentice, programa de Optometría, Fundación Universitaria del Área Andina. mamerchan@hotmail.com

${ }^{3}$ Odontólogo. Especialista en Epidemiología. Docente investigador, posgrados, Facultad de Ciencias de la Salud, Fundación Universitaria del Área Andina. Grupo de investigación Charles Prentice, programa de Optometría, Fundación Universitaria del Área Andina. jmejia2@areandina.edu.co 


\section{PHYTAGORAS HYPOTENUSE AS INDEX OF AMETROPIA}

Gabriel Merchán Mendoza', María Susana Merchán Price², Jairo Emilio Mejía Argüello ${ }^{3}$

\section{Abstract}

General Objective. To create a suitable Index, based on Pythagoras' hypotenuse, to represent any refractive state and to facilitate statistical analyses of ametropic patients. Methods. 3964 patients from a database of OPTILASER (Refractive Surgery Center in Bogotá, Colombia) for the year 2014 were selected to demonstrate the applicability of the proposed Index of Ametropia. The patients had not been previously subjected to any kind of surgical procedure. Another example to illustrate the applicability of the proposed Index of Refraction comes from a database of an investigation "Madre Canguro Integral" (Integral Kangaroo Mother), carried out between 2013 and 2014 at Hospital San Ignacio, Bogotá in which 311 healthy prematurely-born babies were evaluated Principal meridians of ametropias are taken as catheti of a right triangle in order to calculate the Hypotenuse which will be taken as the Index of Ametropia. Spearman Correlation, between the Index of Ametropia and the Visual Acuity expressed as the Minimum Angle of Resolution (MAR), are determined. Results. Spearman Correlation between the Index of Ametropia and the Minimum Angle of Resolution is 0,834 statistically significant ( $\mathrm{p}:<0,001$ ) ample enough to validate the proposed Index of Ametropia. Conclusions. The Index of Ametropia is a valid instrument for the assessment, management and statistical analysis of ametropias. 


\section{A HIPOTENUSA DE PITÁGORAS COMO ÍNDICE DE AMETROPIA}

Gabriel Merchán Mendoza', María Susana Merchán Price², Jairo Emilio Mejía Argüello ${ }^{3}$

\section{Resumo}

Objetivo geral. Criar um índice, baseado na hipotenusa de Pitágoras, para representar qualquer estado refrativo e facilitar a análise estatística de pacientes com ametropia. Métodos. 3964 pacientes de um banco de dados do OPTILASER (Centro de Cirurgia Refrativa de Bogotá, Colômbia) em 2014, foram selecionados para demonstrar a aplicabilidade do Índice de Ametropia proposto. Os pacientes não tinham sido submetidos a nenhum tipo de procedimento cirúrgico. Outro exemplo para ilustrar a aplicabilidade do Índice de Ametropia, vem da pesquisa "Mãe Canguru Integral", realizada entre 2013 e 2014, no Hospital de San Ignacio, Bogotá, onde 311 bebês nascidos prematuramente e com boa saúde foram avaliados. Os meridianos principais da ametropia são tomados como catetos de um triângulo retângulo para calcular a hipotenusa que será tomada como o índice de ametropia. Em seguida, foi determinada a correlação de Spearman entre o Índice de Ametropia e o Ângulo Visual expresso com o Ângulo Mínimo de Resolução (Mínimo Ángulo de Resolución, MAR). Resultados. A correlação de Spearman entre o Índice de Ametropia e o Ângulo Mínimo de Resolução é de 0,834 , o que é estatisticamente significativo $(\mathrm{p}<0,001)$ e suficiente para validar o Índice proposto. Conclusões. $\mathrm{O}$ índice de ametropia é um instrumento válido para a avaliação, manejo e análise estatística das ametropia. 


\section{Introducción}

La investigación epidemiológica en el campo de la salud visual se esfuerza constantemente en encontrar nuevos conocimientos y nuevos métodos de análisis basados en estudios realizados sobre poblaciones de personas. Los datos recogidos de tales estudios deben ser susceptibles de análisis, con las herramientas estadísticas apropiadas y asumiendo que tales datos son confiables.

Medir una ametropía y su agudeza visual en la práctica general usualmente provee suficiente información para establecer un diagnóstico del estado refractivo, incluyendo la severidad de los síntomas y la disposición apropiada del paciente. Cuando se trata de estudios epidemiológicos, la situación es muy diferente por cuanto es necesario manejar grandes cantidades de datos refractivos. Las ametropías se consideran esféricas cuando los meridianos principales tienen el mismo poder refractivo. De otra manera, se consideran ametropías astigmáticas. En el caso de ametropías esféricas, sus magnitudes proveen suficiente información para estimar su severidad y poder diagnosticar y prescribir los lentes correctivos correspondientes. Sin embargo, este no es el caso en ametropías astigmáticas. Astigmatismos de, por ejemplo, 2,50 dioptrías, no proveen suficiente información, puesto que esta cifra solamente representa la diferencia entre los meridianos principales, pero falla en identificar la naturaleza y magnitud de la ametropía subyacente.

Las ametropías esféricas son fácilmente clasificadas como bajas, medianas o altas, independientemente si son miópi- cas o hipermetrópicas. Las astigmáticas, por el contrario, se clasifican de muchas maneras: localización de los meridianos principales en relación con la retina; localización, vertical u horizontal, del meridiano más afectado; la frecuencia de la orientación del astigmatismo en la población general; la calidad de la superficie corneal y la armonía entre los dos ojos.

En cualquier campo de la ciencia, la investigación puede requerir variadas herramientas de análisis, tales como descripción de variables, comparación de muestras, análisis de varianza, correlaciones, regresiones, test $Z$, test $T$, etc.(1). Cualquiera que sea el caso, se requiere claridad y definición precisa de los datos. Es necesario, por tanto, tener un índice o indicador que compendie en un número único y en cualquier estado refractivo, esférico o astigmático, así como que correlacione todas las variables con la severidad. Además, sirve como guía para definir las conductas generales de salud visual que puedan aplicarse a la población en general.

De acuerdo con el Free Dictionary, "un índice es un número resultado de una fórmula, que se usa para caracterizar un conjunto de datos para poder derivar conclusiones" (2).

Existen muchos índices en medicina para evaluar diferentes tipos de situaciones. Por ejemplo, está el Apache I y II para clasificación de las enfermedades, el triage como guía en unidades de emergencia (UCI) para cuidado médico especial, evaluación de desastres y clasificación médica de enfermedades espe- 
cíficas según su severidad. Todos ellos se basan en mediciones fisiológicas objetivas, independientemente de las guías terapéuticas, y su propósito es maximizar la efectividad de los cuidados de los pacientes y disminuir el desperdicio de esfuerzos y recursos.

El uso apropiado de la estadística en ametropías antes y después de cualquier intervención ha sido siempre un problema. La combinación de esferas y cilindros es más compleja de lo que generalmente se cree por cuanto el componente cilíndrico de las ametropías astigmáticas solo representa una diferencia en dioptrías entre los meridianos principales y no una entidad ametrópica como la miopía o la hipermetropía. Algunas de las alternativas actualmente usadas por los investigadores para sus análisis estadísticos son las siguientes:

Recientemente, Merchán y Merchán(3) clasificaron las ametropías de acuerdo con su severidad mediante un criterio puramente clínico. La severidad se interpretó como el grado en el que un error refractivo afecta la capacidad de las personas para funcionar en la vida cotidiana. Las categorías de ametropías son susceptibles de análisis estadístico por medios no-paramétricos.

Holladay(4) pretendió evaluar la relación entre error refractivo, agudeza visual y tamaño pupilar en pacientes intervenidos de queratotomía radial. Para ello, propuso el desenfoque equivalente, que consiste en sumar el valor absoluto (+) de la mitad del cilindro y el equivalente esférico absoluto en casos de miopía (-) o hipermetropía (+) bajo cicloplejia. Holladay solo da un ejemplo en su desenfoque equivalente.

Por otro lado, en 1980, Keating propone el uso de matrices para sumar lentes esfero-cilíndricos en los casos de pacientes que usan prescripciones que necesitan ser modificadas por medio de procedimientos de sobrerefracción(5).

Posteriormente, en 1983, ilustra el "uso de matrices para obtener el valor promedio de los errores refractivos"(6) haciendo énfasis sobre las ventajas de la matriz poder-dióptrico, que juega el mismo papel en lentes esfero-cilíndricos que el papel del poder-dióptrico en lentes esféricos. Sus ecuaciones permiten convertir esferocilindros en matrices que, a su vez, pueden ser sumadas y promediadas. En 1988, Harris emplea las ecuaciones de Long y muestra el efecto quirúrgico refractivo de varios procedimientos quirúrgicos por medio de matrices que representan esfero-cilindros(7). También menciona, pero no discurre, la posibilidad del análisis estadístico. En 1991, Long desarrolla matrices para representar las esferas, cilindros y ejes de lentes convencionales y obtener así una esfera equivalente(8).

En 2002, Kaye describe cómo el álgebra matricial puede representar esfero-cilindros y errores refractivos $y$, de esta forma, sumar, restar, multiplicar, invertir, y elevar a potencias los esfero-cilindros(9). Por su parte, Thibos fundamenta sus investigaciones sobre el análisis de Fourier de los tres elementos del astigmatismo(10).

Las esferas, cilindros y ejes de múltiples 
que se someten a análisis estadísticos $y$, finalmente, se reconvierten a su forma esfera, cilindro y eje. Miller sigue la misma aproximación para ilustrar cómo obtener una refracción promedio de múltiples ametropías(11). Gartner provee las fórmulas matemáticas para calcular vectores de cilindros(12). Raasch correlaciona la agudeza visual con la longitud vectorial de los esfero-cilindros y encuentra una correlación significativa entre miopía esférica, astigmatismo miópico compuesto $\mathrm{y}$ astigmatismo mixto(13).

\section{Propósito}

Calcular un índice de ametropía que facilite el manejo estadístico de los estados refractivos, con base en el teorema de Pitágoras.

\section{Materiales y métodos}

Cuando los meridianos principales del ojo se toman como los lados de un triángulo rectángulo, se puede determinar la hipotenusa de tal triángulo. Esta hipotenusa representará al estado refractivo del ojo mediante un número único que incluya todas las posibles variaciones de la refracción ocular, el cual llamamos índice de refracción. Para validarlo, es indispensable que tenga una correlación significativa con la agudeza visual, dado que las ametropías son la causa principal del emborronamiento de las imágenes en la retina (desenfoque). Teniendo en cuenta que las fracciones de Snellen no son expresiones matemáticas reales(8), la agudeza visual Snellen ha sido reemplazada por el log del mínimo angulo de resolución (LogMAR), que ha sido universalmente aceptado. si el índice de ametropía aumenta, el LogMAR aumenta y la agudeza visual disminuye.

En la Figura 1 se puede observar un triángulo rectángulo, en el cual $a$ y $b$ se denominan lados o catetos y $c_{2}$ la hipotenusa. Los lados son independientes y mutuamente perpendiculares, por lo tanto, la magnitud de un lado no afecta la magnitud del otro. La hipotenusa, por el contrario, no es independiente y su magnitud está determinada por la de ambos lados.

Esta característica de la hipotenusa es precisamente la razón para asimilarla como hipotenusa de refracción. Esta también depende, en modo similar, de los independientes meridianos principales de la refracción ocular que son mutuamente perpendiculares. 
Figura 1. Triángulo rectángulo, a y b son los catetos y c, la hipotenusa

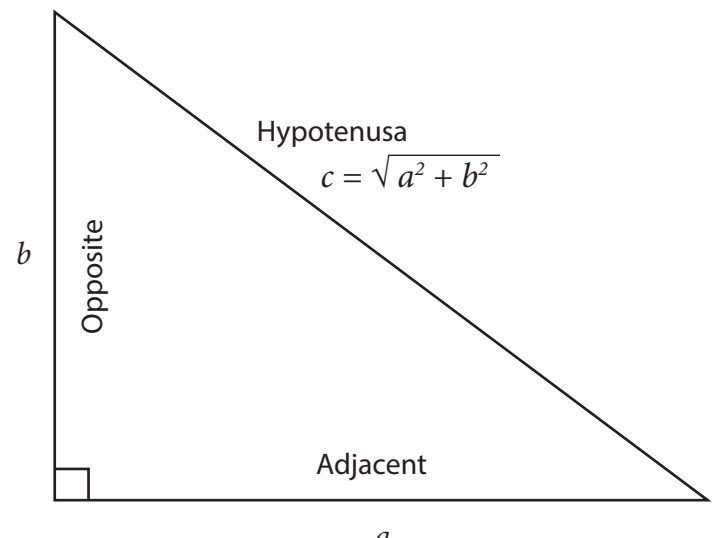

Fuente: Elaboración propia.

El teorema de Pitágoras dice simplemente que el cuadrado de la hipotenusa es igual a la suma de los cuadrados de los catetos.

$$
\begin{gathered}
\text { Hipotenusa }^{2}=\text { lado } \mathrm{a}^{2}+\text { lado } \mathrm{b}^{2} \\
\text { Por lo tanto, Hipotenusa }=\sqrt{ }\left(\text { lado } \mathrm{a}^{2}+\text { lado } \mathrm{b}^{2}\right)
\end{gathered}
$$

Si aplicamos el mismo principio a la refracción ocular, podemos escribir: índice de ametropía $=\sqrt{ }\left(\right.$ Meridiano Primario $^{2}+$ Meridiano Secundario $\left.^{2}\right)$

$$
\text { Ejemplo: } \mathrm{Rx}:+3,00 \text { esf }\left(-1,25 \text { cil eje } 0^{\circ}\right)
$$

meridiano primario: $+3,00$ Dpt. meridiano secundario: $+1,75 \mathrm{Dpt}$.

$$
\text { índice de ametropía }=\sqrt{ }\left(+3,00^{2}+1,75^{2}\right)=\mathbf{3 , 4 7}
$$

Dado que el índice de ametropía se determina sumando el cuadrado de cada meridiano principal, lo cual los hace positivos, seguido de la raíz cuadrada del total, es irrelevante que los datos refractivos provengan de la fórmula de los lentes correctores o de los poderes refractivos del error de refracción.

En relación con el eje del cilindro, debe tenerse claro que no tiene incidencia en la severidad de las ametropías y no hay necesidad de incluirlo en los estudios estadísticos de los estados refractivos. Un eje a $180^{\circ}$ no es más importante que uno a $60^{\circ}$. El eje solo es importante cuando el objeto de interés está formado por componentes horizontales, verticales u oblicuos, tales como letras impresas o edificaciones urbanas. De otra forma, no es importante cuando observamos paisajes $\mathrm{u}$ otros objetos exentos de elementos lineales. Es obvio que el eje tiene un papel clave en la prescripción de lentes para corregir 
Para demostrar la aplicabilidad del índice de ametropía propuesto, se seleccionaron 3964 pacientes de una base de datos de Optiláser (Centro de cirugía refractiva en Bogotá, Colombia) elaborada en el 2014. Los pacientes no habían sido sometidos previamente a ningún procedimiento quirúrgico. Las evaluaciones optométricas estuvieron a cargo de Carmen Cecilia Villa, optómetra de la institución.

Otro ejemplo para ilustrar la aplicabilidad del índice de ametropía propuesto viene de una base de datos de 311 bebés en buena salud, nacidos prematuramente y evaluados en el programa Madre Canguro Integral entre 2013 y 2014, en el Hospital San Ignacio de Bogotá. El objetivo general de la investigación fue describir el desarrollo de bebés prematuros, se obtuvieron los valores normativos del test basado en la escala de Bayley para el desarrollo de infantes lactantes, y el test basado en la escala de Griffiths para el desarrollo mental a los 3, 6, 9 y 12 meses de edad gestacional corregida.

Como parte de tal investigación, la refracción optométrica fue realizada a los 3 y a los 9 meses para buscar posibles correlaciones entre el desarrollo general y el desarrollo visual del niño. Es aquí donde el índice de ametropía puede usarse para determinar la media, la mediana, la moda y la desviación estándar de las ametropías involucradas a los 3 y a los 9 meses de edad.

Metodológicamente, la presente investigación es cuantitativa, descriptiva y correlacional.

\section{Resultados}

La Tabla 1 y la Tabla 2 muestran la estadística descriptiva para el índice de ametropía y LogMAR. La asimetría y curtosis del índice de ametropía claramente muestran una distribución normal, mientras la asimetría y curtosis para LogMAR indican una distribución casi normal. Consecuentemente, la correlación entre estas variables deben ser examinadas por medio de rho de Spearman en lugar de la $r$ de Pearson. Correlaciones superiores a 0,4 se consideran altas. En nuestro caso, rho es 0,834, de modo que indica una correlación muy alta (casi perfecta). $\mathrm{R}^{2}$ es 0,695 lo que significa que el $69,5 \%$ de los hallazgos LogMAR están directamente relacionados con el índice de ametropía. El $30,5 \%$ restante se atribuye a la acomodación ejercida por los hipermétropes, entre otros factores. 
Tabla 1. Estadísticas de la muestra

\begin{tabular}{lccc}
\hline & & $\begin{array}{c}\text { Índice de } \\
\text { ametropía }\end{array}$ & LogMAR \\
\hline Valid & Lost & 3964 & 3963 \\
Media & 3981 & 3982 \\
Mediana & 4,4196 &, 7477 \\
Moda & 3,3600 &, 6020 \\
Desviación estándar &, 25 &, 89 \\
Asimetría & 4,09374 &, 52961 \\
Error estándar asimetría & $\mathbf{2 , 5 0 9}$ &, $\mathbf{6 9 5}$ \\
Kurtosis & $\mathbf{2}$ &, 039 \\
Error estándar de la Kurtosis & $\mathbf{9 , 5 4 4}$ &, $\mathbf{2 4 7}$ \\
\hline
\end{tabular}

Fuente: Elaboración propia.

Tabla 2. Correlación entre índice de ametropía y LogMAR

\begin{tabular}{|c|c|c|c|c|}
\hline & & & $\begin{array}{l}\text { Índice de } \\
\text { Ametropía }\end{array}$ & LogMAR \\
\hline \multirow{6}{*}{ Spearman Rho } & \multirow{3}{*}{ Índice de Ametropía } & $\begin{array}{l}\text { Coeficiente de } \\
\text { correlación }\end{array}$ & 1,000 &, 834 \\
\hline & & Sig. (bilateral) & &, 000 \\
\hline & & N & 3964 & 3963 \\
\hline & \multirow[t]{3}{*}{ LogMAR } & $\begin{array}{l}\text { Coeficiente de } \\
\text { correlación }\end{array}$ &, 834 & 1,000 \\
\hline & & Sig. (bilateral) &, 000 & \\
\hline & & $N$ & 3963 & 3963 \\
\hline
\end{tabular}

Fuente: Elaboración propia. 
Figura 2. Regresión: índice de ametropía y LogMAR

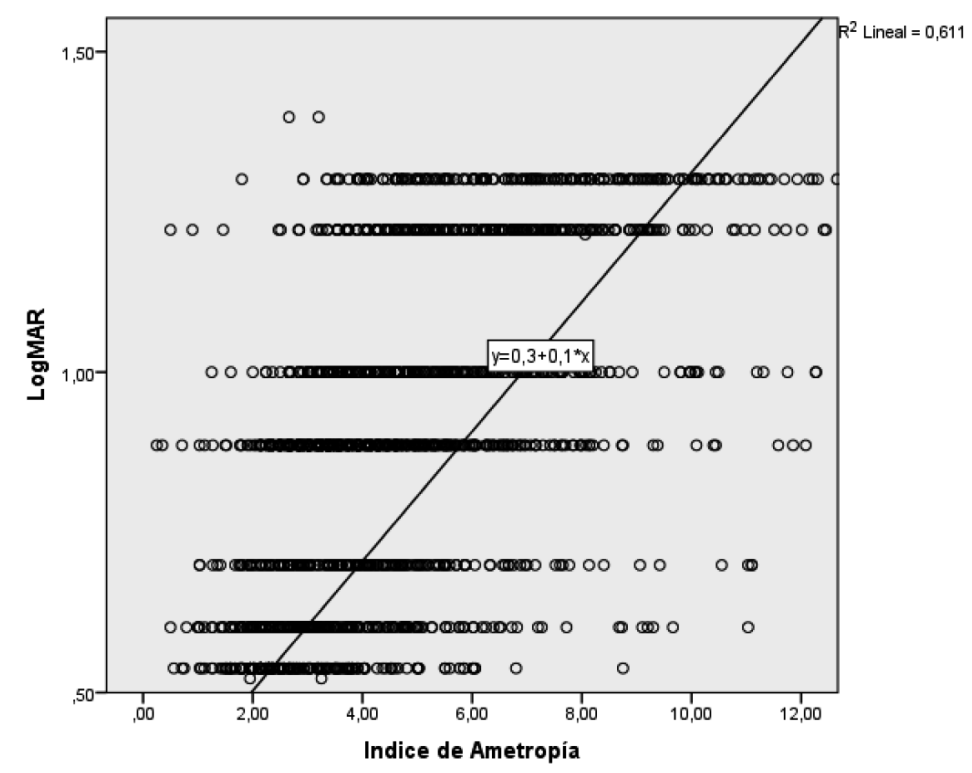

Fuente: Elaboración propia.

Una comparación entre el índice de ametropía y el método de vectores se muestra en la Tabla 3 y Tabla 4, relacionadas con la evolución del estado refractivo en bebés prematuros a edades 3 meses (294 ojos) y 9 meses ( 329 ojos). La primera columna, índice de ametropía, muestra la media, la mediana, la moda y la desviación estándar de los datos. La segunda columna muestra las mismas funciones, convirtiendo previamente la notación clínica a vectores $(\mathrm{C} 2 \mathrm{~V})$, de acuerdo con Miller(11). En la tercera columna, vemos la reconversión de vectores de regreso a notación clínica (V2C), cilindro positivo y en la columna final la notación clínica con cilindro negativo.

Tabla 3. Comparación entre índice de ametropía y vectores, 3 meses de edad

\begin{tabular}{lllllllllllll}
\hline Índice de ametropía & \multicolumn{4}{c}{ C2V } & \multicolumn{3}{c}{ V2C (+Cilindro) } & \multicolumn{3}{c}{ V2C (-Cilindro) } \\
\hline Media & 3,15 & 1,60 & 0,80 & 0,00 & 0,80 & 1,60 & 90 & 2,40 & $-1,60$ & 180 \\
Mediana & 3,00 & 1,50 & 1,00 & 0,00 & 0,50 & 2,00 & 90 & 2,50 & $-2,00$ & 180 \\
Moda & 2,83 & 1,00 & 1,00 & 0,00 & 0,00 & 2,00 & 90 & 2,00 & $-2,00$ & 180 \\
$\mathbf{7 2}$ & $\begin{array}{l}\text { Desviación } \\
\text { estándar }\end{array}$ & 1,41 & 1,66 & 0,43 & 0,00 & 1,77 & 0,82 & 90 & 1,65 & $-0,82$ & 180 \\
\hline
\end{tabular}

Fuente: Elaboración propia. 
Tabla 4. Comparación entre índice de ametropía y vectores, 9 meses de edad

\begin{tabular}{lc|cccccccccc}
\hline \multicolumn{1}{l}{ Índice de ametropía } & \multicolumn{3}{c}{ C2V } & \multicolumn{3}{c}{ V2C (+Cilindro) } & \multicolumn{3}{c}{ V2C (-Cilindro) } \\
\hline Media & 1,90 & 0,86 & $-0,61$ & 0,00 & 0,24 & 1,23 & 90 & 1,47 & $-1,23$ & 180 \\
Mediana & 1,58 & 1,00 & $-0,50$ & 0,00 & 0,50 & 1,00 & 90 & 1,50 & $-1,00$ & 180 \\
Moda & 1,00 & 1,00 & $-0,50$ & 0,00 & 0,50 & 1,00 & 90 & 1,50 & $-1,00$ & 180 \\
$\begin{array}{l}\text { Desviación } \\
\text { estándar }\end{array}$ & 1,38 & 1,27 & 0,35 & 0,00 & 0,92 & 0,70 & 90 & 1,62 & $-0,70$ & 180 \\
\hline
\end{tabular}

\section{Discusión}

Aunque el índice de ametropía no tiene una representación óptico-anatómica, como el equivalente esférico o el desenfoque equivalente, sí tiene el soporte matemático de los meridianos principales del ojo, de la misma forma en que los lados del triángulo rectángulo definen la hipotenusa. Adicionalmente, el índice de ametropía que proponemos supera los métodos utilizados actualmente para compendiar y manejar los datos refractivos destinados al análisis estadístico. Estos métodos fueron ya mencionados en la sección introductoria pero un cor- to análisis de sus desventajas puede ser útil en este punto.

Equivalente esférico: su mayor desventaja es que no distingue entre cilindros altos y bajos, lo cual es clínica y estadísticamente inconveniente. Además, un equivalente esférico dado puede representar varios defectos refractivos. Si promediamos 6 y 4 , el promedio es 5 . El mismo resultado entre 7 y 3,8 y 2,9 y 1 o 4 y 6.

La Tabla 5 ilustra el punto con ametropías positivas y negativas. La columna 5 muestra el índice de ametropía de cada ametropía y la columna 6 el correspondiente equivalente esférico.

Tabla 5. Comparación entre índice de ametropía y equivalente esférico

\begin{tabular}{c|c|c|c|c|c}
\hline $\begin{array}{c}\text { Esfera } \\
\text { meridiano } \\
\text { Primario }\end{array}$ & Cilindro & Eje & $\begin{array}{c}\text { Meridiano } \\
\text { secundario }\end{array}$ & $\begin{array}{c}\text { Índice de } \\
\text { ametropía }\end{array}$ & $\begin{array}{c}\text { Equivalente } \\
\text { esférico }\end{array}$ \\
\hline-1.75 & -0.50 & $0^{\circ}$ & -2.25 & 2.85 & -2.00 \\
2.50 & -1.00 & $0^{\circ}$ & 1.50 & 2.92 & 2.00 \\
-1.00 & -2.00 & $90^{\circ}$ & -3.00 & 3.16 & -2.00 \\
3.25 & -2.50 & $90^{\circ}$ & 0.75 & 3.34 & 2.00 \\
3.75 & -3.50 & $0^{\circ}$ & 0.25 & 3.76 & 2.00 \\
0.00 & -4.00 & $90^{\circ}$ & -4.00 & 4.00 & -2.00 \\
5.00 & -6.00 & $0^{\circ}$ & -1.00 & 5.10 & 2.00 \\
\hline
\end{tabular}

Fuente: Elaboración propia. 
Los índices de ametropía se muestran ordenadamente para reflejar la gravedad creciente de las ametropías. El equivalente esférico, por otra parte, no tiene significación porque refleja solamente los signos positivos y negativos de las refracciones miópicas e hipermetrópicas. Sin embargo, no hay indicaciones sobre la severidad de los síntomas causados por los varios estados refractivos.

Sistema de vectores: el eje del astigmatismo no debe ser considerado en el análisis por vectores porque su posición no afecta la severidad de las ametropías. Los ejes a $50^{\circ}$ no son más severos que a $20^{\circ}$. El índice de ametropía propuesto no toma en cuenta el eje del cilindro, evitando así cálculos matemáticos innecesarios.

Categorización de ametropías: las categorías de ametropías son susceptibles de manejo estadístico, pero solamente por métodos no-paramétricos.

Desenfoque equivalente: Holladay(4) no especifica el manejo matemático para sumar el valor absoluto $(+)$ del cilindro al equivalente esférico en casos de miopía (-) o hipermetropía (+). Además, las desventajas del equivalente esférico, ya mencionadas, se aplican igualmente al desenfoque equivalente. El índice de ametropía evita cálculos innecesarios y actúa solamente sobre los meridianos de poder de la refracción ocular.

De otra parte, el índice de ametropía propuesto excede a los métodos actuales que compendian y manejan los datos refractivos para su análisis estadístico, basándonos en los siguientes criterios:

- Es un número único que toma en consideración los estados refracti- vos de los meridianos principales y desecha los ejes de los cilindros astigmáticos.

- Se ocupa principalmente de la visión a distancia, aunque puede extrapolarse para visión próxima mediante algunos cálculos matemáticos.

- Es susceptible de análisis estadístico descriptivo e inferencial.

Como se ve en las Tablas 3 y 4, el método de Miller(11), basado en las fórmulas de Long(8) y usadas también por Harris(9) y Thibos(10), puede producir refracciones promedio, medianas promedio, modas promedio y desviaciones estándar promedio. Esto se logra cuando se convierte la notación clínica (esfera, cilindro y eje) en vectores separados ( $M$, J0 y J45) para finalmente convertir los vectores de regreso a notación clínica. No hay objeciones desde el punto de vista matemático. Sin embargo, no es fácil juzgar o clasificar los resultados en términos de severidad por la simple razón que no se especifica un índice de severidad. ¿Sobré cuál componente debemos basar nuestros criterios para estimar la severidad de los resultados? ¿La esfera, el cilindro o el eje?

Las Tablas 3 y 4 corresponden con un estudio para determinar la evolución del proceso de emetropización del estado refractivo en neonatos prematuros a las edades de 3 y 6 meses. La primera columna de las tablas muestra el promedio, la mediana, la moda y la desviación estándar del índice de ametropía. Es fácil observar los cambios refractivos con una simple ojeada, mientras que el método de vectores no indica claramente si corresponden a la esfera, el cilindro o el eje. Una mirada más cuidadosa muestra 
la diferencia entre los promedios (medias) de los índices de ametropía: a los 3 meses, 3,15; a los 9 meses, 1,90, lo que significa que la ametropía a los 3 meses es considerablemente mayor que a los 9 meses y esto corresponde con el bien conocido proceso de emetropización.

Creemos que el estado refractivo no debe descomponerse entre esfera, cilindro y eje. Es difícil discernir cuál componente refleja más exactamente la severidad del estado refractivo. Podríamos preguntarnos cuál indica mayor severidad, si una esfera de 4 dioptrías o un cilindro de 4 dioptrías. Nuestro índice de ametropía se hace cargo de los estados refractivos, desechando cilindros y ejes y tomando en su lugar, los meridianos principales.

Con todo, debemos considerar la situación en la que es deseable conocer la ametropía, esférica o astigmática, que yace detrás de un índice de ametropía dado. La manera en la que se concibe el índice de ametropía no permite que se vaya en reversa y que se encuentre la ametropía de la cual fue derivado. En otras palabras, conocer solamente la hipotenusa de un triángulo rectángulo no hace posible que se determinen los catetos que la originaron. Esto no es necesariamente una desventaja, puesto que, en epidemiología, es la gravedad de las secuelas causadas por un estado refractivo defectuoso lo que verdaderamente importa.

Parece más lógico llevar el índice de ametropía a una escala de severidad, como se muestra en la Tabla 6. El índice de ametropía obtenido en alguna investigación epidemiológica permite concluir, posiblemente, que el grupo bajo escrutinio puede necesitar la atención de autoridades locales o estatales para corregir cualquiera que sea el factor, o factores, que están actuando sobre la población (iluminación, nutrición, ambiente, etc.). Adicionalmente, el índice de ametropía permite el análisis comparativo de muestras provenientes de distintas poblaciones o de una misma población en tiempos diferentes.

Tabla 6. Escala de severidad del índice de ametropía.

Tabla 6. Escala de severidad del índice de ametropía.

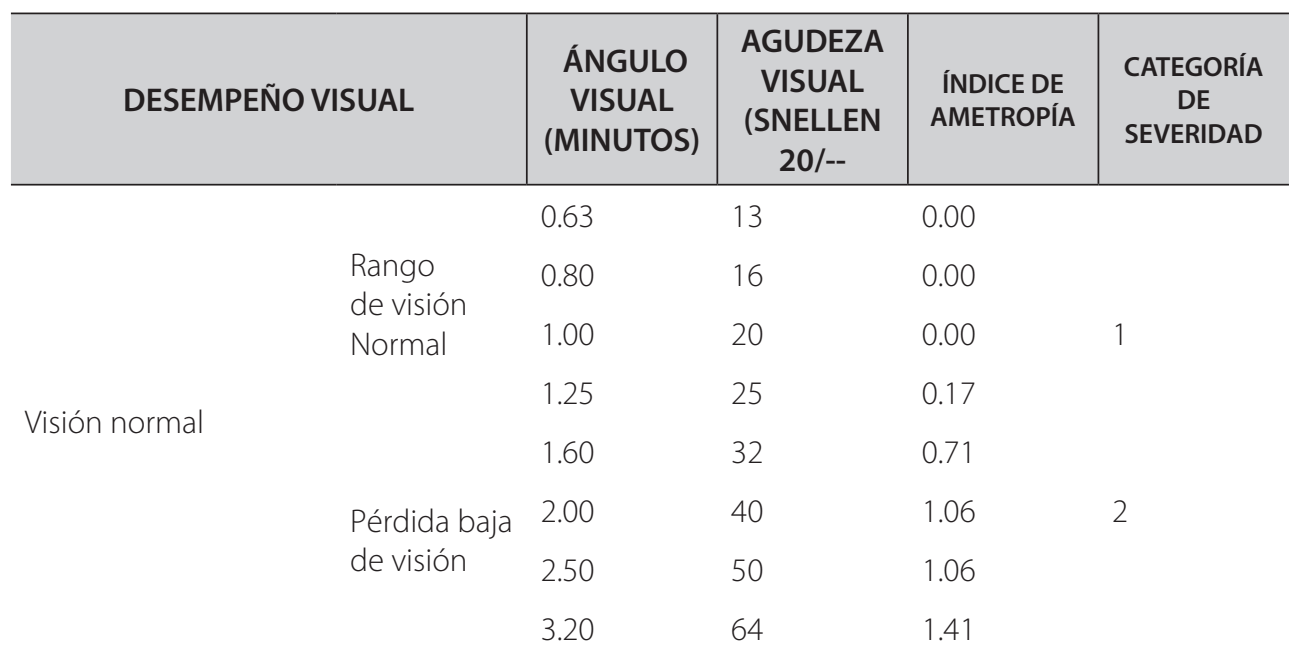




\begin{tabular}{|c|c|c|c|c|c|}
\hline \multicolumn{2}{|c|}{ DESEMPEÑO VISUAL } & $\begin{array}{l}\text { ÁNGULO } \\
\text { VISUAL } \\
\text { (MINUTOS) }\end{array}$ & $\begin{array}{c}\text { AGUDEZA } \\
\text { VISUAL } \\
\text { (SNELLEN } \\
20 /--\end{array}$ & $\begin{array}{c}\text { ÍNDICE DE } \\
\text { AMETROPIÍA }\end{array}$ & $\begin{array}{c}\text { CATEGORÍA } \\
\text { DE } \\
\text { SEVERIDAD }\end{array}$ \\
\hline \multirow{12}{*}{ Baja Visión } & \multirow{4}{*}{$\begin{array}{l}\text { Pérdida } \\
\text { moderada } \\
\text { de visión }\end{array}$} & 4.00 & 80 & 1.77 & \multirow{5}{*}{3} \\
\hline & & 5.00 & 100 & 2.12 & \\
\hline & & 6.30 & 126 & 2.48 & \\
\hline & & 8.00 & 160 & 2.83 & \\
\hline & & 10.00 & 200 & 3.00 & \\
\hline & \multirow{4}{*}{$\begin{array}{l}\text { Pérdida } \\
\text { severa de } \\
\text { visión }\end{array}$} & 12.50 & 250 & 3.89 & \multirow[t]{4}{*}{4} \\
\hline & & 16.00 & 320 & 4.60 & \\
\hline & & 20.00 & 400 & 5.47 & \\
\hline & & 25.00 & 500 & 6.53 & \\
\hline & \multirow{4}{*}{$\begin{array}{l}\text { Pérdida } \\
\text { profunda de } \\
\text { visión }\end{array}$} & 32.00 & 640 & 7.43 & \multirow[t]{3}{*}{5} \\
\hline & & 40.00 & 800 & 8.84 & \\
\hline & & 50.00 & 1000 & 10.25 & \\
\hline \multirow{4}{*}{ Cercano a ceguera } & & 63.00 & 1260 & Cuenta & \multirow{4}{*}{6} \\
\hline & \multirow[t]{2}{*}{ Casi ciego } & 80.00 & 1600 & dedos & \\
\hline & & 100 & ------ & ------ & \\
\hline & Ceguera & NLP & NLP & NLP & \\
\hline
\end{tabular}

Resumiendo, el índice de ametropía que condensa los meridianos de la refracción ocular en un número único es una herramienta valiosa en la investigación epidemiológica del cuidado visual por facilitar la aplicación de análisis estadísticos descriptivo e inferenciales.

\section{Conclusiones}

La correlación de Spearman en $\mathbf{0 , 8 3 4}$ entre el índice de ametropía y el LogMAR, es estadísticamente significativa (p: $<\mathbf{0 , 0 0 1 )}$. Por ello, concluimos que el índice de ametropía es un indicador válido para estimar la severidad de ametropías, tanto esféricas como astigmáticas. En consecuencia, es útil para análisis estadísticos cuantitativos. La $\mathrm{r}^{2}$ obtenida indica que el $62,56 \%$ de las variaciones del LogMAR están directamente relacionadas con variaciones en el índice de ametropía. El 37,43\% restante depende de otros factores, como la acomodación en los hipermétropes, edad, tamaño pupilar y estado general de salud, entre otros. Finalmente, el índice de ametropía es una herramienta adecuada para categorizar las ametropías de acuerdo con la severidad de los síntomas visuales y el grado de impedimentos causados. 


\section{Reconocimientos}

\section{Fundación Universitaria del Área}

Andina.

Optiláser, optómetra Pablo Henao De

Brigard,

Optómetra María Victoria Báez,

Optómetra Carmen Cecilia Villa.

Doctor Luis Humberto Molina por su asesoría estadística.

\section{Referencias}

1. Horton N, Hardin J. Teaching the Next Generation of Statistics Students to "Think with Data": Special Issue on Statistics and the Undergraduate Curriculum. Am Stat. 2015 nov;69(4):259

2. The Free Dictionary by Farlex. https:// es.thefreedictionary.com/

3. Merchán $M$, Merchán $G$, Dueñas $M$. Influencia de la prematuridad en el proceso de emetropización. Rev Pediatr. 2014;47:83-9.

4. Holladay J, et al. The relationship of visual acuity, refractive error, and pupil size after radial keratotomy. Arch Ophthalmol. 1991;109:70-6.

5. Keating M. An easier method to obtain the sphere, cylinder, and axis from an off-axis dioptric power matrix. Am J Optom and Physiol Opt. 1980;57:734-7.

6. Keating M. On the use of matrices for the mean value of refractive errors. $A m$ Ophthal and Physiol Opt.1983;3:201-3.

7. Harris W. Algebra of sphero-cylinders and refractive errors, and their means, variance, and standard deviation. $A m \mathrm{~J}$ Optom Physiol Opt.1988;65:794-802.

\section{Financiamiento}

El proyecto fue financiado por la Fundación Universitaria del Área Andina.

\section{Conflictos de interés}

Los autores no tienen conflictos de interés que declarar.

8. Long W. Lens power matrices and the sum of equivalent spheres. Optom Vis Sci. 1991;68:821-2.

9. Kaye SB, Harris WF. Analyzing refractive data. J Cataract Refract Surg. 2002; 28: 2109-2116.

10. Thibos L, Horner D. Power vectors: an application of Fourier analysis to the description and statistical analysis of refractive error. Optom Vis Sci. 1997;74:367-75.

11. Miller J. Clinical applications of power vectors. Optom Vis Sci. 2009;86:599-602.

12. Gartner W. Astigmatism and optometric vectors. Optom Vis Sci. 1965;42:459-63.

13. Raasch T. Spherocylindrical refractive errors and visual acuity. Optom Vis Sci. $1995 ; 72: 272-5$.

14. Espinosa J, Merchán P. Estudio retrospectivo del estado refractivo en niños prematuros de tres a cuatro meses de edad corregida, realizado en el programa Madre Canguro Integral, Hospital San Ignacio, Bogotá. Cienc Tecnol Salud Vis Ocul. 2012;10:11-21. 



\section{PHYTAGORAS HYPOTENUSE AS INDEX OF AMETROPIA}

Gabriel Merchán Mendoza', María Susana Merchán Price², Jairo Emilio Mejía Argüello 3

\section{Abstract}

General Objective. To create a suitable Index, based on Pythagoras' hypotenuse, to represent any refractive state and to facilitate statistical analyses of ametropic patients. Methods. 3964 patients from a database of OPTILASER (Refractive Surgery Center in Bogotá, Colombia) for the year 2014 were selected to demonstrate the applicability of the proposed Index of Ametropia. The patients had not been previously subjected to any kind of surgical procedure. Another example to illustrate the applicability of the proposed Index of Refraction comes from a database of an investigation "Madre Canguro Integral" (Integral Kangaroo Mother), carried out between 2013 and 2014 at Hospital San Ignacio, Bogotá in which 311 healthy prematurely-born babies were evaluated Principal meridians of ametropias are taken as catheti of a right triangle in order to calculate the Hypotenuse which will be taken as the Index of Ametropia. Spearman Correlation, between the Index of Ametropia and the Visual Acuity expressed as the Minimum Angle of Resolution (MAR), are determined. Results. Spearman Correlation between the Index of Ametropia and the Minimum Angle of Resolution is 0,834 statistically significant ( $\mathrm{p}:<0,001$ ) ample enough to validate the proposed Index of Ametropia. Conclusions. The Index of Ametropia is a valid instrument for the assessment, management and statistical analysis of ametropias. 


\section{LA HIPOTENUSA DE PITÁGORAS COMO ÍNDICE DE AMETROPÍA}

Gabriel Merchán Mendoza', María Susana Merchán Price², Jairo Emilio Mejía Argüello 3

\section{Resumen}

Objetivo General. Crear un Índice, con base en la hipotenusa de Pitágoras, para representar cualquier estado refractivo y facilitar los análisis estadísticos de los pacientes amétropes. Métodos. 3964 pacientes de una base de datos de OPTILASER (Centro de Cirugía Refractiva en Bogotá, Colombia) del año 2014, fueron seleccionados para demostrar la aplicabilidad del Índice de Ametropía propuesto. Los pacientes no habían sido sometidos a ningún tipo de procedimiento quirúrgico. Otro ejemplo para ilustrar la aplicabilidad del Índice de Ametropía, viene de la investigación "Madre Canguro Integral" llevada a cabo entre 2013 y 2014 en el Hospital de San Ignacio, Bogotá, en la cual 311 bebés nacidos prematuramente y en buen estado de salud, fueron evaluados. Los Meridanos Principales de las ametropías se toman como catetos de un triángulo rectángulo para calcular la Hipotenusa que será tomada como Índice de Ametropía. Seguidamente, se determinó la correlación de Spearman entre el Índice de Ametropía y el Ángulo Visual expresado con Mínimo Angulo de Resolución (MAR). Resultados. La correlación de Spearman entre el Índice de Ametropía y el Mínimo Ángulo de Resolución es 0,834 lo cual es estadísticamente significativa $(\mathrm{p}:<0,001)$ y suficiente para validar el Índice propuesto. Conclusiones. El Índice de Ametropía es un instrumento válido para la evaluación, manejo y análisis estadístico de las ametropías. 


\section{LA HIPOTENUSA DE PITÁGORAS COMO ÍNDICE DE AMETROPÍA}

Gabriel Merchán Mendoza', María Susana Merchán Price², Jairo Emilio Mejía Argüello 3

\section{Resumo}

Objetivo geral. Criar um índice, com base na hipotenusa de Pitágoras, para representar qualquer estado de refração e facilitar a análise estatística dos pacientes ametrópico. Métodos. 3964 pacientes de um OPTILASER banco de dados (Centro de Cirurgia Refrativa em Bogotá, Colômbia) em 2014, foram selecionados para demonstrar a aplicabilidade da proposta Índice Ametropia. Os pacientes não foram submetidos a nenhum tipo de procedimento cirúrgico. Outro exemplo para ilustrar a aplicabilidade do Índice Ametropia, vem da pesquisa "Mãe Canguru Integral", realizado entre 2013 e 2014, o Hospital de San Ignacio, Bogotá, onde 311 bebês nascidos prematuramente e com boa saúde,eles foram avaliados. Principais Meridanos de ametropia são tidos como as pernas de um triângulo retângulo para calcular a hipotenusa para ser tomado como índice Ametropia. Em seguida, foi determinada a correlação de Spearman entre o Índice de Ametropia e o Ângulo Visual expresso com o Ângulo de Resolução Mínima (MARM). Resultados. A correlação de Spearman entre a ametropia e índice de ângulo mínimo de resolução é de 0,834 , que é estatisticamente significativa $(\mathrm{p}<0,001)$ e suficiente para validar o índice proposto. Conclusões. O índice de ametropia é um instrumento válido para a avaliação, gestão e análise estatística da ametropia. 


\section{Introduction}

Epidemiological research, in the field of visual health, strives continuously to find new knowledge and new methods of analysis based on studies performed on populations of individuals. The data collected from such studies should be susceptible of analysis with the appropriate statistical tools on the assumption that such data are trustworthy.

Ametropias are considered spherical when the Principal Meridians have the same refractive power, otherwise they are considered astigmatic ametropias. In the case of spherical Ametropias, their magnitudes provide sufficient information to appraise its severity and diagnose and prescribe the corresponding correcting lenses. This is not the case in astigmatic ametropias. An astigmatism of, say, 2,50 diopters, does not provide enough information since it only represents the difference between the Principal Meridians but fails to identify the nature and magnitude of the underlying Ametropia.

Spherical ametropias are easily classified as low, median and high, regardless of whether they are myopic or hyperopic. Astigmatic ametropias, on the contrary, are classified in many ways such as location of principal meridians relative to the retina; the location, vertical or horizontal, of the most affected meridian; the frequency of a given orientation of astigmatism in the general population; the quality of the corneal surface, and the harmony of astigmatisms in the two eyes.
In clinical practice, measuring an Ametropia and its visual acuity, usually constitutes enough information to establish diagnosis of the refractive state including severity of symptoms and proper disposal of patient. A very different situation arises when dealing with epidemiological studies in which you must handle large numbers of refractive data.

Any research on any field of science, may need several tools for analysis such as description of variables, comparison of samples, variance analysis, correlations, regressions, $\mathrm{Z}$ tests, $\mathrm{t}$ tests, etc. ${ }^{1}$ Whatever the case, clarity and precise definition of data and tools are required. It is necessary, therefore, to have an Index or Indicator, that condenses in a single number, any refractive state, spherical o astigmatic, and summarizes all variables to severity and serves as guide to define proper clinical and general visual health actions that can apply to the general population.

According to The Free Dictionary " $a n$ Index is a number, that results from a formula, that is used to characterize an array of data and permits drawing conclusions". ${ }^{2}$

There are many indexes in medicine to evaluate diverse types of situations, e.g.: Apache I and II for disease classification, Triage guide for Hospital Emergency Units, ICU patient care, evaluation of disasters, medical classification for specific diseases according to severity. All these indexes are based on objective physiological measurements independently of therapeutic guidelines and they are intended to maximize the effectiveness 
of patient care and, to diminish wasted efforts and resources.

The proper use of statistics in ametropias, before and after any intervention, has always been a problem. The combination of "spheres" and "cylinders" is more complex than generally thought since the cylindrical component of astigmatic ametropias represents a dioptric difference between the principal meridians and not an ametropic entity like myopia or hyperopia. Some of the alternatives currently used by researchers to perform statistical analyses are the following:

Recently, Merchán and Merchán ${ }^{3}$, classified ametropias into categories according to severity with a purely clinical criterion. Severity was taken as the degree by which a refractive error jeopardizes the ability of a person to function in everyday life. Categories of Ametropias are amenable to statistical analyses by non-parametric methods.

Holladay $^{4}$, pretended to evaluate the relation between refractive error, visual acuity and pupil size, in patients after radial keratotomy. For that purpose, He proposed the Defocus Equivalent consisting of adding the absolute value (+) of half the cylinder to the absolute Spherical Equivalent in case of myopia (-) or hyperopia (+) under cycloplegia. He only gives one example of his DE. Keating $^{5}$ in 1980, deals with the use of matrices for adding spherocylindrical lenses in the case of a patient wearing some prescription that needs to be modify by over-refraction procedure.
Later, Keating ${ }^{6}$ in 1983, illustrates the "use of matrices for the mean value of refractive errors" emphasizing the advantage of the dioptric power matrix which plays the same role for spherocylindrical lenses as the dioptric power plays for spherical lenses. His equations allow to convert sphero-cylinders into matrices which, in turn, can be added and averaged. Harris, in $1988^{7}$, using Long's equations, shows the Refractive Surgical Effect of various surgical procedures by means of matrices to represent sphero-cylinders. He also mentions, but does not dwell into, the possibility of statistical analyses.

Long ${ }^{8}$, in 1991, develops matrices to represent sphere, cylinder and axis of conventional prescription lenses and adding the traces of two or more power matrices, the equivalent sphere is obtained. Kaye $^{9}$ later in 2002, describes how matrix algebra can represent sphero-cylinders and refractive errors and in so doing, sphero-cylinders can be added, subtracted, multiplied, inverted and raised to powers.

Thibos $^{10}$ based their investigation on Fourier analysis of the three elements of astigmatism. Spheres, cylinders and axes of multiple ametropias are converted into power vectors that are then, subjected to statistical analyses to finally convert the results back into sphere, cylinder and axis notation. Miller,11 follows the same approach to illustrate how to obtain an average refraction from multiple cases of ametropias. Gartner12, provides the mathematical formulae to calculate cylinder vectors. Raasch13, correlates visual acuity with the vector 
length of sphero-cylinders and found a significant correlation between spherical myopia, compound myopic astigmatism and mixed astigmatism.

\section{Purpose}

To calculate.the Index of Ametropia to facilitate the statistical handling of refractive states based on Pythagoras Theorem.

\section{Material and Methods}

When the Principal Meridians of the eye are taken as legs of a right triangle, the hypotenuse of such triangle can be determined. Such hypotenuse will represent the refractive state of the eye with a single number that embraces all possible variations of ocular refraction. We call it Index of Ametropia. To validate it, it must have a significant correlation to Visual Acuity since ametropias are the principal cause of blurring of images on the retina (defocus). Considering that Snellen fractions are not real mathematical expressions, ${ }^{8}$ Snellen Visual Acuity has been substituted by the widely used Log of the Minimum Angle of Resolution (LogMAR). If the Index of Ametropia increases, LogMAR increases and visual acuity decreases.

In the illustration (Fig. 1) we see a right triangle in which $\mathbf{a}$ and $\mathbf{b}$ are called the sides (catheti) and $c$ the hypotenuse. The sides are independent and perpendicular from each other, thus, the magnitude of one side does not affect the magnitude of the other. The hypotenuse on the contrary, is not independent and its magnitude is determined by the magnitude of both sides. This characteristic of the hypotenuse is precisely the reason to assimilate it as Hypotenuse of Refraction which is also dependent, in a similar fashion, to the independent Principal Meridians of the ocular refraction perpendicular to each other.

Figure 1 Right Triangle. a and b are the sides (catheti) and $\mathrm{c}$ is the Hypotenuse.

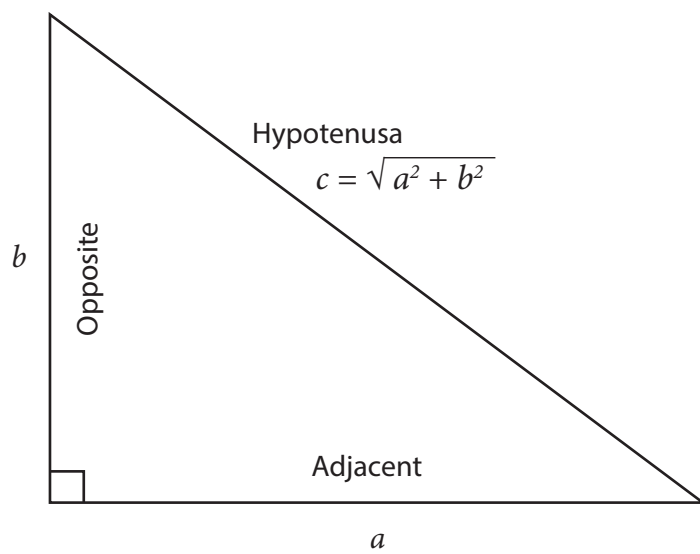

Pythagoras Theorem simply states that the square of the hypotenuse is equal to the sum of the squares of the sides. 
Hypotenuse $^{2}=$ side $\mathrm{a}^{2}+$ side $\mathrm{b}^{2}$

Thus, $\quad$ Hypotenuse $=\sqrt{ }\left(\right.$ side $\mathrm{a}^{2}+$ side $\left.\mathrm{b}^{2}\right)$

Applying the same principle to the ocular refraction, we can write:

Hypotenuse of Refraction ${ }^{2}=$ Primary Meridian $^{2}+$ Secondary Meridian ${ }^{2}$

and Hypotenuse of Refraction $=\sqrt{ }\left(\right.$ Primary Meridian ${ }^{2}+$ Secondary Meridian $\left.^{2}\right)$

Example: Given: Rx: $+3.00 \mathrm{sph}\left(-1.25 \mathrm{cyl}\right.$ axis $\left.0^{\circ}\right)$, thus,

Primary Meridian: +3.00 Dpts. Secondary Meridian: +1.75 Dpts.

Index of Ametropia $=\sqrt{ }(+3.002+1.752)=3,47$

Since the Index of Ametropia is determined by adding the square of each of the Principal Meridians, which turns them all positive, followed by the square root of the total, it becomes irrelevant whether refractive data comes from the spectacle correction of the Ametropia or from the dioptric powers of the refractive error. Hyperopic and myopic ametropias have the same Index of Ametropia since both have the same defocus and the same circle of diffusion provided accommodation is at rest.

In relation to the cylinder Axis, it must be said that it has no bearing on the severity of ametropias and need not be included in statistical studies of visual and refractive conditions. An axis at $180^{\circ}$ is no more significant than axis at $60^{\circ}$. The axis becomes important only when the object of interest is formed by horizontal, vertical or oblique components, such as print letters or city buildings. Otherwise, it is not important when observing landscapes or other objects devoid of linear elements. However, it does have a key role in prescribing lenses to correct astigmatic defects.

3964 patients from a database of OPTILASER (Refractive Surgery Center in Bogotá Colombia) for the year 2014 were selected to demonstrate the applicability of the proposed Index of Ametropia. The patients had not been previously subjected to any kind of surgical procedure. Optometric evaluations were conducted by Carmen Cecilia Villa, optometrist of the Institution.

Another example to illustrate the applicability of the proposed Index of Refraction comes from a database of an investigation "Madre Canguro Integral" (Integral Kangaroo Mother), carried out between 2013 and 2014 at Hospital San Ignacio, Bogotá in which 311 healthy prematurely-born babies were evaluated. The general Objective was to describe the development of premature babies by obtaining the normative values for a test based on Bayley scales for development of breast-feeding infants (3rd edition) and a test based on Griffiths scales of mental development (revised edition) at 3, 6, 9 and 12 months corrected gestational age.

As part of such investigation, optometric refraction was performed at 3 and 9 months to find possible relationships between whole development and visual development of the child. It is, at this point, where the Index of Ametropia can 
mode and standard deviation of the ametropias involved at 3 and 9 months of age.

Type of Statistical Analysis: Quantitative, descriptive and correlational.

\section{Results}

Descriptive Statistics for the Index of Ametropia and LogMAR are shown in Table 1 and table 2. Skewness and Kurtosis for Index of Ametropia clearly show an abnormal distribution whereas
Skewness and Kurtosis for LogMAR indicate nearly normal distribution. Consequently, correlation between those variables must be tested by Spearman's rho rather than Pearson's r. Correlations higher than 0,4 are considered high. In our case rho is 0,834 indicating a very high correlation (near perfect). $\mathbf{R}^{2}$ is 0,695 meaning that $69,5 \%$ of LogMAR findings are directly correlated to Index of Ametropia. The remaining 30,5\% shows the influence of accommodation exerted by hyperopes, among other factors.

Table 1 Statistics of the sample

\begin{tabular}{|c|c|c|c|}
\hline & & Index of Ametropia & LogMAR \\
\hline \multirow{2}{*}{$\mathrm{N}$} & Valid & 3964 & 3963 \\
\hline & Lost & 3981 & 3982 \\
\hline Media & & 4,4196 & ,7477 \\
\hline Median & & 3,3600 & 6020 \\
\hline Mode & &, 25 &, 89 \\
\hline Standard Deviation & & 4,09374 & ,52961 \\
\hline Asimetry & & 2,509 & 695 \\
\hline Asimetry standard error & &, 039 &, 039 \\
\hline Kurtosis & & 9,544 & ,247 \\
\hline Standard error of Kurtosis & & ,078 & ,078 \\
\hline
\end{tabular}

Table 2 Correlation between Index of Ametropia and LogMAR

\begin{tabular}{|c|c|c|c|c|}
\hline & & & $\begin{array}{c}\text { Índice de } \\
\text { Ametropía }\end{array}$ & LogMAR \\
\hline \multirow{6}{*}{ Spearman Rho } & \multirow{3}{*}{ Index of Ametropía } & $\begin{array}{l}\text { Correlation } \\
\text { coefficient }\end{array}$ & 1,000 &, 834 \\
\hline & & Sig. (bilateral) & &, 000 \\
\hline & & $\mathrm{N}$ & 3964 & 3963 \\
\hline & \multirow[t]{3}{*}{ LogMAR } & $\begin{array}{l}\text { Correlation } \\
\text { coefficient }\end{array}$ &, 834 & 1,000 \\
\hline & & Sig. (bilateral) &, 000 & \\
\hline & & $\mathrm{N}$ & 3963 & 3963 \\
\hline
\end{tabular}


Figure 2. Regression: Index of Ametropia and LogMar

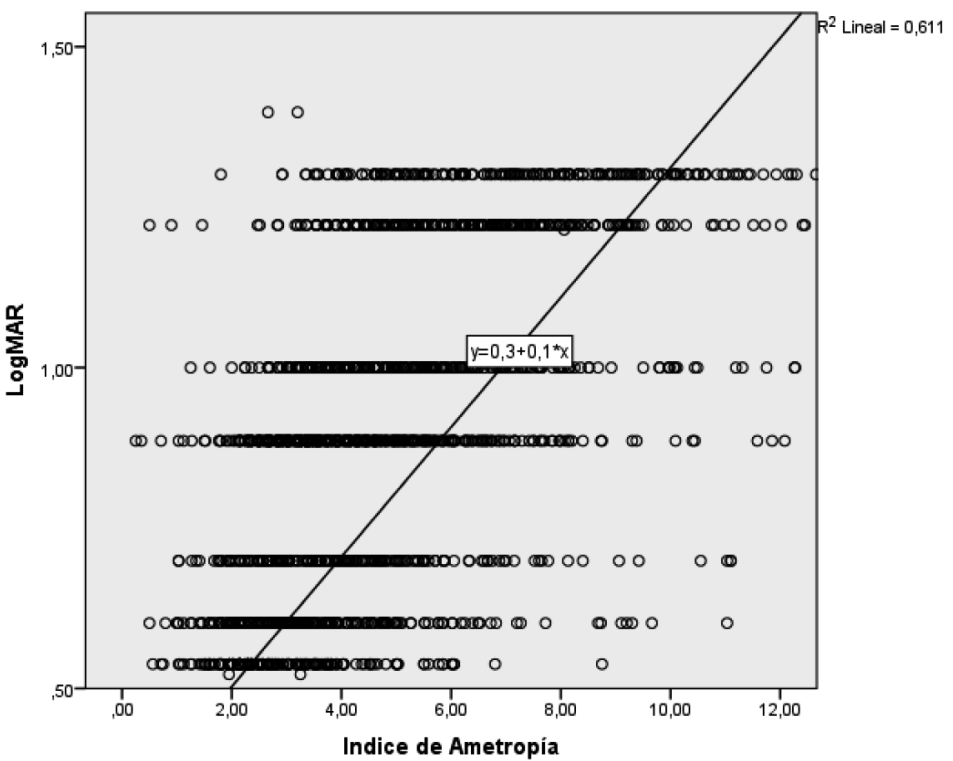

A comparison between the Index of Ametropia and Power Vector methods is shown in Table 3 and 4 which deals with the evolution of refractive state in premature babies at ages 3 months (294 eyes) and 9 months (329 eyes). The first column, Index of Ametropia, shows the results for media, median, mode and standard deviation of the data (data not shown). The second column shows results of the same functions, previous conversion of the data clinical notation to power vectors $(\mathrm{C} 2 \mathrm{~V})$ according to Miller11. In the third column we see the re-conversion of power vectors back to clinical notation (V2C), positive cylinder, and the final column, the negative cylinder clinical notation.

Table 3 Comparison between Index of Ametropia and Power Vectors at ages 3 months

\begin{tabular}{lcccccccccc}
\multicolumn{2}{c|}{ Index of Ametropia } & \multicolumn{3}{c}{ C2V } & \multicolumn{3}{c|}{ V2C (+Cylinder) } & \multicolumn{3}{c}{ V2C (-Cylinder) } \\
\hline Media & 3,15 & 1,60 & 0,80 & 0,00 & 0,80 & 1,60 & 90 & 2,40 & $-1,60$ & 180 \\
Median & 3,00 & 1,50 & 1,00 & 0,00 & 0,50 & 2,00 & 90 & 2,50 & $-2,00$ & 180 \\
Mode & 2,83 & 1,00 & 1,00 & 0,00 & 0,00 & 2,00 & 90 & 2,00 & $-2,00$ & 180 \\
Standard Deviation & 1,41 & 1,66 & 0,43 & 0,00 & 1,77 & 0,82 & 90 & 1,65 & $-0,82$ & 180 \\
\hline
\end{tabular}

Table 4 Comparison between Index of Ametropia and Power Vectors at 9 months

\begin{tabular}{lc|cccccccccc}
\hline \multicolumn{2}{c|}{ Index of Ametropia } & \multicolumn{3}{c|}{ C2V } & \multicolumn{3}{c|}{ V2C (+Cylinder) } & \multicolumn{3}{c}{ V2C (-Cylinder) } \\
\hline Media & 1,90 & 0,86 & $-0,61$ & 0,00 & 0,24 & 1,23 & 90 & 1,47 & $-1,23$ & 180 \\
Median & 1,58 & 1,00 & $-0,50$ & 0,00 & 0,50 & 1,00 & 90 & 1,50 & $-1,00$ & 180 \\
Mode & 1,00 & 1,00 & $-0,50$ & 0,00 & 0,50 & 1,00 & 90 & 1,50 & $-1,00$ & 180 \\
Standard Deviation & 1,38 & 1,27 & 0,35 & 0,00 & 0,92 & 0,70 & 90 & 1,62 & $-0,70$ & 180 \\
\hline
\end{tabular}




\section{Discussion}

Although the proposed Index of Ametropia does not have an optical-anatomical representation, like the Spherical Equivalent or the Defocus Equivalent, it does have the mathematical support of the Principal Meridians of the eye in the same manner that the sides of a right triangle define the hypotenuse.

These methods were already mentioned in the Introductory section, but a short analysis of their shortcomings could be helpful at this point.
Spherical Equivalent. Its main drawback consists of not differentiating between high and low cylinders which is clinically and statistically inconvenient. Furthermore, a given Spherical Equivalent may represent several refractive states. If we average 6 and 4, the average is 5 , the same is obtained with 7 and 3; 8 and $2 ; 9$ and 1 or 4 and 6 .

Table 5 illustrates this point with positive and negative ametropias. Column 5 shows the Index of Ametropia of each Ametropia and column 6 shows the corresponding Spherical Equivalent.

Table 5 Comparison between Index of Ametropia and Spherical Equivalent.

\begin{tabular}{c|c|c|c|c|c}
\hline Sphere Primary & Cylinder & Axis & Secondary & $\begin{array}{c}\text { Index } \\
\text { Ametropia }\end{array}$ & $\begin{array}{c}\text { Spherical } \\
\text { Equivalent }\end{array}$ \\
\hline-1.75 & -0.50 & $0^{\circ}$ & -2.25 & 2.85 & -2.00 \\
2.50 & -1.00 & $0^{\circ}$ & 1.50 & 2.92 & 2.00 \\
-1.00 & -2.00 & $90^{\circ}$ & -3.00 & 3.16 & -2.00 \\
3.25 & -2.50 & $90^{\circ}$ & 0.75 & 3.34 & 2.00 \\
3.75 & -3.50 & $0^{\circ}$ & 0.25 & 3.76 & 2.00 \\
0.00 & -4.00 & $90^{\circ}$ & -4.00 & 4.00 & -2.00 \\
5.00 & -6.00 & $0^{\circ}$ & -1.00 & 5.10 & 2.00 \\
\hline
\end{tabular}

The Indexes of Ametropia are shown in orderly fashion that reflect the increasing severity of the ametropias. The Spherical Equivalent, on the other hand, becomes meaningless reflecting only the plus and minus signs for the corresponding hyperopic and myopic refractions but there is no indication of severity of symptoms that arise in the various states of refraction.

System of Vectors. The axis of astigmatism should not be considered in vector analysis because axes locations do not represent severity in ametropias. Axes at $50^{\circ}$ are no more severe than axes at $20^{\circ}$. The proposed Index of Ametropia does not take cylinder axis avoiding unnecessary mathematical calculations.

Categorization of Ametropias. Categories of ametropias are amenable to statistical analyses but only by non-parametric methods. The Index of Ametropia allows any kind of statistical analysis.

Defocus Equivalent. Holladay4 does not specify the mathematical handling 
of adding the absolute value $(+)$ of the cylinder to the Spherical Equivalent in case of myopia (-) or hyperopia (+). Furthermore, the drawbacks of the Spherical Equivalent apply equally to the Defocus Equivalent. The Index of Ametropia avoids unnecessary calculations and acts solely on the power meridians of the ocular refraction.

Furthermore, the proposed Index of Ametropia excels the current methods of summarizing and handling refractive data for statistical analyses and is based on the following criteria:

- It is a single number that takes into consideration the refractive state of the principal meridians without regard to the astigmatic cylinder.

- It concerns itself mainly with distant vision although it might be extrapolated for near vision with a little mathematical juggling.

- It is amenable to descriptive and inferential statistical analyses.

As shown in Table 3 and 4, Miller's11 method (based on Long's8 formulae, also used by Harris 9 and Thibos10) can produce "average refractions", "median refractions", "mode refractions" and "standard deviation refractions". This is achieved by converting the clinical notation (Sphere, Cylinder and Axis) into separate vectors (M, J0 and J45) and finally convert the vectors back into clinical notation. Mathematically speaking, there is no objection. However, it is not easy to judge or qualify, the results in terms of severity for the simple reason that the index of severity has not been specified. On which component should we base our criterions for appraising the severity of the results: the sphere? the cylinder? the axis?

Tables 3 and 4 correspond to a study to determine the emmetropization evolution of the refractive state in premature newborns at ages 3 and 9 months. First column of the tables shows the Index of Ametropia for mean, median, mode and standard deviation. It is easy to observe at a glance, the refractive changes whereas, the power vector method does not clearly indicate whether the significant refractive changes correspond to the sphere, the cylinder or the axis. Furthermore, a closer look at Tables 3 and Table 4 shows the difference between the media of IA at 3 months $(3,15)$ is much higher than at 9 months $(1,90)$ meaning that ametropia at 3 months is also higher than at 9 months. This corresponds to the well-known emmetropization process14.

We believe that the refractive state should not be broken into sphere, cylinder and axis. It makes it difficult to discern which component reflects more accurately the severity of a refractive state. One may ask, which is more severe, a 4,00 Dpt. Sphere or a 4,00 Dpt. Cylinder. Our Index of Ametropia takes care of refractive states by disregarding the cylinders and axes and taking into consideration the two principal meridians instead.

Another important aspect has to do with visual care epidemiological studies. The proposed Index of Ametropia can be used to calculate all the descriptive statistics needed for such purpose (me- 
dia, median, mode, standard deviation, variance, correlations, anova, etc.) and the inferential analyses that may be required.

Nevertheless, we must consider a situation in which it is desirable to estimate the Ametropia, spherical or astigmatic, behind a given Index of Ametropia. The Index of Ametropia as it has been conceived, does not lend itself to go backwards to find the ametropia from which it was derived. In other words, given only the hypotenuse, it is not possible to determine the sides that originated it. However, this is not necessarily a disadvantage since in epidemiology, it is the graveness of the sequalae caused by a defective refractive state, that really matters.

It seems more logical to take the Index of Ametropia to a Severity Scale Table 6, the Index of Ametropia obtained in some epidemiological research, to conclude, possibly, that the group under scrutiny, may require the attention of local or state authorities to correct whatever factor is acting on the population (illumination, nutrition, environment, etc.). In addition, the Index of Ametropia allows the comparative analysis of samples from different populations or, same population at various times.

Summarizing, the Index of Ametropia that encompasses the power meridians of the ocular refraction into a single number, is a valuable tool in epidemiological visual health research facilitating the application of descriptive and analytical statistics.

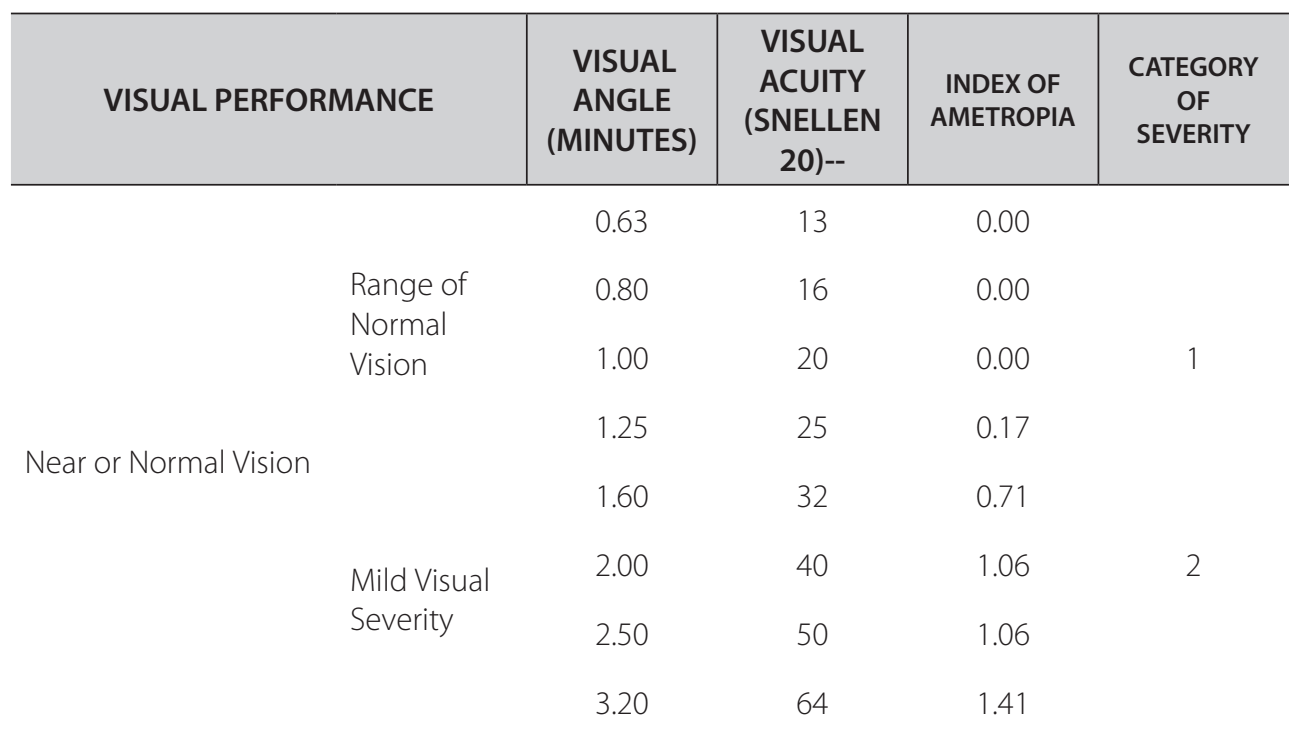




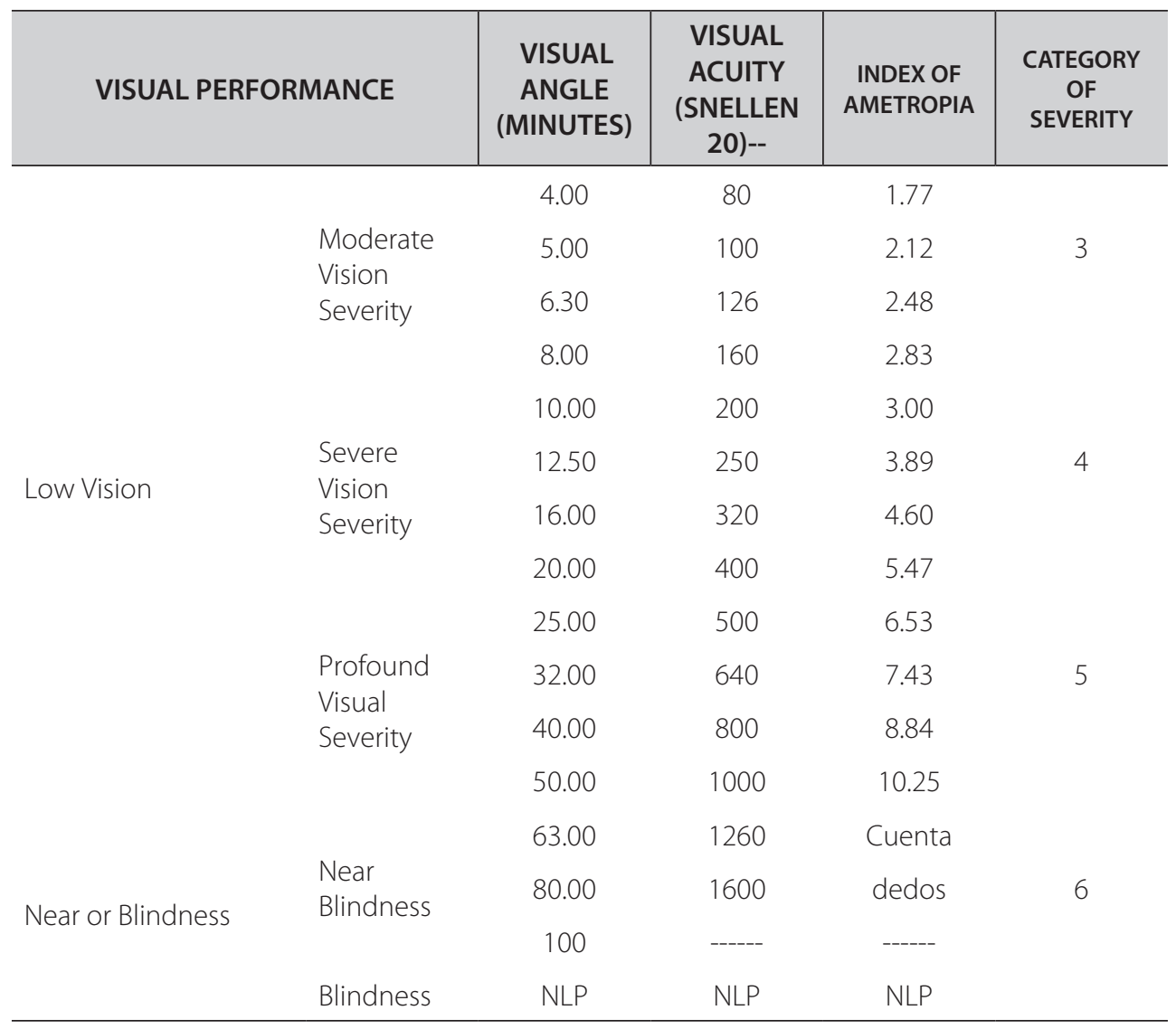

\section{Conclusions}

Spearman Correlation of 0,834 between Index of Ametropia and M.A.R., is statistically significant ( $\mathrm{p}:<0,001)$ and allows us to conclude that the Index of Ametropia is a valid indicator for assessing the severity of both, spherical or astigmatic ametropias and consequently lends itself for proper quantitative and statistical analyses. The $\mathrm{r} 2$ obtained, indicates that $62,56 \%$ of variations of M.A.R. are directly related to variations in the Index of Ametropia. The remaining $37,43 \%$ depends on other factors such as accommodation in hyperopes, age, pupil size and general health. In addition, Index of Ametropia and is an adequate tool for categorizing ametropias in accordance to the severity of the visual symptoms and degree of impairment caused thereby.

\section{Aknowledgements}

Fundación Universitaria del Área Andina OPTILASER, optometrist Pablo Henao De Brigard,

Optometrist María Victoria Báez, Optometrist Carmen Cecilia Villa.

Doctor Luis Humberto Molina for his statistical counseling.

\section{Funding}

This project was funded by an internal 


\section{Conflicts of interest}

The authors have no conflicts of interest to declare.

\section{Bibliography}

1. Horton N, Hardin J. Teaching the Next Generation of Statistics Students to "Think with Data": Special Issue on Statistics and the Undergraduate Curriculum. Am Stat. 2015; 69 Issue 4

2. The Free Dictionary by Farlex. https:// es.thefreedictionary.com/

3. Merchán M, Merchán G, Dueñas M. Influencia de la prematuridad en el proceso de emetropizacion. Rev Pediatr. 2014; 47: 83 - 89 .

4. Holladay JT, Lynn MS, Waring III G, Gemmil M, Keen G, Brook F. The relationship of visual acuity, refractive error, and pupil size after radial keratotomy. Arch Ophthalmol. 1991; 109: 70-76.

5. Keating MP. An easier method to obtain the sphere, cylinder, and axis from an off-axis dioptric power matrix. Am J Optom and Physiol Opt. 1980; 57: 734-737.

6. Keating MP. On the use of matrices for the mean value of refractive errors. Am Ophthal and Physiol Opt. 1983; 3: 201-203.

7. Harris W. F. Algebra of sphero-cylinders and refractive errors, and their means, variance, and standard deviation. Am J Optom Physiol Opt. 1988; 65: 794-802.
8. Long WF. Lens power matrices and the sum of equivalent spheres. Optom Vis Sci. 1991; 68: 821-822.

9. Kaye SB, Harris WF. Analyzing refractive data. J Cataract Refract Surg. 2002; 28: 2109-2116.

10. Thibos LW, Horner D. Power vectors: an application of Fourier analysis to the description and statistical analysis of refractive error. Optom Vis Sci. 1997; 74: $367-375$.

11. Miller JM. Clinical applications of power vectors. Optom Vis Sci. 2009; 86:599-602.

12. Gartner WF. Astigmatism and optometric vectors. Optom Vis Sci. 1965; 42: 459-463.

13. Raasch TW. Spherocylindrical refractive errors and visual acuity. Optom Vis Sci. $1995 ; 72: 272-275$.

14. Espinosa JMS, Merchán PMS. Estudio retrospectivo del estado refractivo en niños prematuros de tres a cuatro meses de edad corregida, realizado en el programa Madre Canguro Integral, Hospital San Ignacio, Bogotá. Cienc Tecnol Salud Vis Ocul. 2012; 10:11-21. 